\title{
Protein Array Technology: The Tool to Bridge Genomics and Proteomics
}

\author{
Holger Eickhoff ${ }^{1} \cdot$ Zoltán Konthur $^{2} \cdot$ Angelika Lueking $^{2} \cdot$ Hans Lehrach $^{2}$. \\ Gerald Walter $^{2} \cdot$ Eckhard Nordhoff $^{1} \cdot$ Lajos Nyarsik $^{2} \cdot$ Konrad Büssow $^{2}$ \\ ${ }^{1}$ Scienion AG, Volmerstr.7b, 12489 Berlin, Germany.E-mail: eickhoff@scienion.com \\ ${ }^{2}$ Max Planck Institute of Molecular Genetics, Ihnestrasse 73, 14195 Berlin, Germany
}

The generation of protein chips requires much more efforts than DNA microchips. While DNA is DNA and a variety of different DNA molecules behave stable in a hybridisation experiment, proteins are much more difficult to produce and to handle. Outside of a narrow range of environmental conditions, proteins will denature, lose their three-dimensional structure and a lot of their specificity and activity. The chapter describes the pitfalls and challenges in Protein Microarray technology to produce native and functional proteins and store them in a native and special environment for every single spot on an array, making applications like antibody profiling and serum screening possible not only on denatured arrays but also on native protein arrays.

Keywords: Protein Array, Expression Profiling, Automation, Serum Screening, Antibody Profiling, Protein Purification

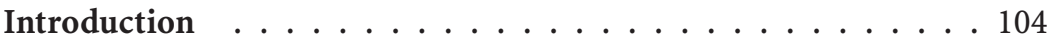

2 From 2D Electrophoresis and Microtitre Plates to Microarrays

of Biomolecules . . . . . . . . . . . . . 105

3 Requirements for Protein Arrays . . . . . . . . . . 106

4 Planar Immobilisation of Proteins _. . . . . . . . . . 107

5 Detection of Molecular Interactions on Microarrays . . . . . . . 108

6 Applications of Protein Arrays . . . . . . . . . . . . . 108

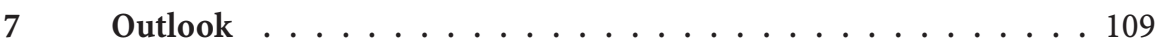

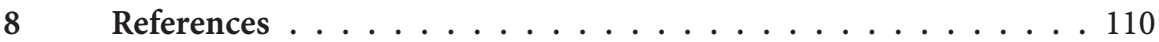


1

\section{Introduction}

Each cell of a living organism contains the whole genetic information in the form of DNA molecules. The available golden path sequence of the human genome is known to be $3 \times 10^{9}$ nucleotide base pairs in size, coding for a currently unknown number of genes. Although the DNA information is, with rare exceptions, identical in each cell, several hundreds of different known cell types do exist. The simplified view of a cell brings up specific populations of DNA and mRNA molecules that are translated into cell-specific populations of proteins. Although this is only a simplified representation, we are currently able to understand only a minority of the complex interactions in the cell machinery comprising DNA, RNA and proteins as major compounds. Genomic databases have enabled us to access, retrieve and process biological information. The determination of the genomic sequences of higher organisms, including humans, is now attainable, but represents only one level of genetic complexity. The determination of the expression profiles and protein profiles of certain cell types represents the next level of genomic complexity, equally important to sequencing.

The interest of our research is focussed in finding all genes, their in vivo functions and the features of the corresponding proteins. Information about a gene's expression is important for its potential exploitation. A gene's expression and the corresponding protein level can be highly specific to a tissue, organ, cell type or disease and, as such, may be attractive as targets for the development of highly specific therapeutics and diagnostics. Even a gene of unknown function may have medical utility if its expression pattern can be determined.

To achieve this goal, methods and technologies operating reliably with many samples in high-throughput and in parallel are major requirements. The human genome is sequenced, but only a minority of genes has been assigned a function. Automated technology allows for high-throughput, resulting in fingerprints of diseased versus normal or developmentally distinct tissues. Differential gene expression can be most efficiently monitored by DNA hybridisation on arrays of oligonucleotides or cDNA clones. Having started from high-density filter membranes, cDNA microarrays nowadays are mainly used in chip or microscope slide format. In the past our group has shown that the same cDNA libraries used in gene expression analysis can be used for highthroughput protein expression and antibody screening on high-density filters and microarrays. Most importantly, these libraries connect recombinant proteins to clones identified by DNA hybridisation or sequencing, hence creating a direct link between gene catalogues and functional catalogues. Microarrays can now be used to go from an individual clone to a specific gene and its protein product or vice versa. Clone libraries have become amenable to database integration including all steps from DNA sequencing to functional assays of proteins.

The medical application of this information is expected to lead to new generations of products in the diagnostics and therapeutics market. However, genes will only be useful for drug development and medical diagnostics if their functions are known. To tackle the current limitations in the medical use of 
genome information, "functional genomics" or "functional proteomics" are now under development as a new research and development area.

With the introduction of automated technologies in the field of molecular biology and, especially, microarray technology, genome and gene expression analysis have been accelerated enormously. Microarray technology was enabled by the development of devices that can array biological samples at high density and with high precision [1]. Oligonucleotide and cDNA microarrays have become hot commodities, representing thousands of individual genes arrayed on filter or glass slide supports (The chipping forecast, Nature Genetics supplement 1999 [2]). To examine variation in gene expression, sets of oligonucleotides or complex probes, generated by reverse transcription of RNA from different tissues and cell lines, are hybridised on the arrays [3]. cDNA microarrays have already been used to profile human tissues like bone marrow, brain, prostate and heart [4] and complex diseases such as rheumatoid arthritis [5] and cancer [6, 7]. However, the DNA chip technology is still hampered by the lack of common quality standards that enable the comparison of results obtained in different laboratories and with arrays of different origin $[8,9]$. Nonetheless, protein chips are already emerging to follow DNA chips as tools for automated and miniaturised functional analysis $[10,11]$. Analogous to DNA microarrays, protein arrays offer the opportunity to screen thousands of immobilised biomolecules at a time, using steadily reduced amounts of sample.

\section{2 \\ From 2D Electrophoresis and Microtitre Plates to Microarrays of Biomolecules}

Two-dimensional gel electrophoresis separates proteins according to size and charge, therefore allowing the study of cell, tissue and even whole organism proteomes [12]. Until recently, however, the identification of the thousands of separated proteins used to be a major challenge. With the introduction of new and automated mass spectrometric protein identification procedures, the high throughput identification of the separated proteins is much simplified [13] and allows us to generate catalogues of expressed proteins in a cell or tissue of interest. Nevertheless, as the separated proteins are obtained in denatured form and in limited amounts, the expression of a protein of interest in recombinant form is usually required for functional characterisation. The classical array format in proteomics, the microtitre plate, is a well established and still widely used standard in medical diagnostics. To increase the number of samples and decrease reagent volume, the 96-well microtitre plate has been developed further to plates with 384 and 1536 wells, maintaining the original plate footprint. As it happened in DNA analysis earlier, the microtitre plate is now gradually being replaced by microarrays on flat surfaces such as glass slides ("chips") or membranes.

The format and the preparation of protein microarrays depends on the nature of the immobilised biomolecule and its application. While peptide arrays are manufactured synthetically directly on the support [14], proteins are delivered using either pin-based spotting or liquid microdispensing. To date, the most com- 
monly arrayed proteins are antibodies, since they are robust molecules which can be easily handled and immobilised by standard procedures without loss of activity.

\section{3}

\section{Requirements for Protein Arrays}

For protein arrays, resources of large numbers of proteins, preferably in purified form, represent a major technical challenge. While in prokaryotes genomic fragments can be directly cloned into expression vectors, intronic sequences prohibit this strategy in eukaryotes. In systems such as human tissues, full-length cDNA clones have to be isolated before protein expression can be started. Highthroughput subcloning of open reading frames has been described [15] but remains a major difficulty if complete proteomes of higher organisms represent the region of interest. To overcome these problems, arrayed cDNA expression libraries, cloned in bacterial and yeast expression vectors, have been developed in our laboratory. These libraries are generated by standard DNA cloning procedures and characterised by oligonucleotide fingerprinting to be screened for the properties of their expression products. Furthermore, these libraries do represent an immortal source for large numbers of recombinant proteins [16-18]. In addition, expression libraries eliminate the need to construct individual expression systems for every protein of interest and, by arraying, the expression products of complete libraries can be characterised in parallel. On the other hand, a large proportion of clones do not express their insert in a suitable form, mainly due to cDNA fragments being fused to the vector-encoded start codon in the wrong reading frame. Therefore, non-expression clones have to be identified and removed from the library. To identify expression clones, hundreds of thousands of clones are arrayed on filter membranes and protein expression is induced. By detection of a $\mathrm{His}_{6}$-tag peptide fused to the protein products, desired expression clones are identified and re-arrayed into a non redundant daughter expression library. The protein filter array technology was further developed to increase spot density and to facilitate the arraying of purified proteins. Lueking et al. have used automated arraying from liquid expression cultures using a pin-based, flat-bed gridding robot [19]. For this purpose, 96 proteins of the human foetal brain cDNA library hEx1 [17] were expressed in liquid bacterial cultures, and solutions were spotted onto polyvinylidene difluoride (PVDF) filters, either as crude lysates or after purification by Ni-NTA immobilised metal affinity chromatography (IMAC). In all 4,800 samples were placed onto polyacrylamide-coated microscopic slides and simultaneously screened, using a hybridisation automat, applying minimal amounts of reagents (less than $100 \mu \mathrm{L}$ antibody solution; A. Lueking, personal communication). Sharp and well-localised signals allowed the detection of 250 attomol or $10 \mathrm{pg}$ of a spotted test protein (GAPDH, glyceraldehyde-3-phosphate dehydrogenase, Swiss-Prot P04406). 


\section{4}

\section{Planar Immobilisation of Proteins}

The Lehrach laboratory mainly uses solid pins routinely for spotting nanolitre volumes of proteins although proteins can be delivered onto solid supports by either split pin-based spotting or microdispensing devices. In contrast to other techniques, solid pins are less sensitive to variation of sample viscosity than slit pins or microdispensing systems and are much easier to clean to prevent any cross contaminations $[19,20]$. A ring and pin arraying device was used by MacBeath and Schreiber to produce a microarray of 10,800 spots of two distinct proteins (protein $\mathrm{G}$ and an FKBP12 binding domain), which were then specifically detected with fluorescently labelled IgG and FKBP12, respectively. Although the detection scheme is this article is really clever, it remains unclear why the authors did array 10800 replicates made of only two proteins [21]. As an alternative to modified glass surfaces gel immobilisation matrices show high binding capacities and provide the proteins with a nearly ( $97 \%$ water in the buffer system) native environment. This is a key feature in protein array research and is important for the reactivity and specificity of the arrayed proteins. The technique of immobilisation is substantial both for effective concentration and orientation of immobilised proteins or antibodies on the surface. A variety of methods have been reported, including the adsorption to charged or hydrophobic surfaces, covalent cross-linking or specific binding via tags (e.g., $\mathrm{His}_{6}$, biotin/avidin system).

The density of protein molecules immobilised on the support is mainly determined by the surface structure. A flat, two-dimensional surface offers less binding capacity than the three-dimensional structure of a filter membrane or a polyacrylamide gel layer. Mirzabekov and co-workers produced three-dimensional polyacrylamide gel pad microarrays providing a more than 100 times greater immobilisation capacity than two-dimensional glass supports, thus increasing the sensitivity of measurements considerably [22]. The gel pads are separated by a hydrophobic glass surface and provide a native, aqueous environment and can accommodate proteins of up to 400,000 Dalton in size [23]. Enzymatic activity of several enzymes like horseradish peroxidase, alkaline phosphatase and $\beta$-D-glucuronidase has been detected in these hydrogel pads. Prestructured surfaces consisting of hydrophilic spots on hydrophobic surfaces have also been reported for protein arraying [24,25]. The hydrophobic surface prevents the aqueous drops applied to the hydrophilic spots from mixing and creates wall-minimised reaction vessels, where the interactions can be monitored in solution. In combination with state of the art microfabrication procedures, prestructured surfaces allow the introduction of three-dimensional microstructures on a chip, offering a number of additional options for experimentation like on-line monitoring of the interaction kinetics. Such microfluidic devices can be equipped with channels for transporting reagents to immobilised target molecules. At the present time microfluidic chips do certainly offer specific advantages over planar microarrays [26] but due to their complex production procedures and the high surface to volume ratio that represents a potential non-specific binding site for the analyte, their development and applications are still at an early stage. 


\section{5}

\section{Detection of Molecular Interactions on Microarrays}

On DNA microarrays, hybridisation events are detected using fluorescently or radioactively labelled probe molecules [27]. A corresponding approach for the detection of protein-protein, protein-DNA and protein-small molecule interactions has been reported, the "universal protein array system" (UPA), consisting of filter membrane arrays of purified proteins [28]. Specific binding properties of the immobilised proteins on the low-density UPA arrays were demonstrated with various radiolabelled protein, DNA, RNA and small molecule ligands. By washing the membrane with different salt conditions, high-affinity protein-protein interactions could be distinguished. In addition to fluorescent dyes and radioisotopes, a wide range of detection options exists for protein and antibody arrays (reviewed in [29]). Unlabelled ligands can be identified indirectly by using a secondary antibody (sandwich assay). Alternatively to these non-competitive formats, various competitive assays, relying on competition of the ligand with labelled tracers, are in use. Protein chips for direct measurement of protein mass by matrix-assisted laser desorption-ionisation time-of-flight (MALDI-TOF) mass spectrometry have been described $[20,24,30]$.

\section{6}

\section{Applications of Protein Arrays}

A large variety of assays has been adapted to utilise protein microarrays. At its current state, the detection of immobilised antigens with antibodies is still the most common application. Protein and antibody arrays have been used for the selection and characterisation of novel antibodies from phage display libraries and for the identification of antigens (e.g., involved in autoimmune diseases).

Phage display antibody libraries have been developed for the in vitro selection of antibodies as an alternative to animal immunisation (reviewed in [32], [53]). For this purpose, recombinant immunoglobulin gene libraries are cloned in phagemid vectors and antibody fragments are displayed as fusion proteins on the surface of bacteriophage (reviewed in [34]). Recently, protein arrays of the cDNA expression library hEx1 [17] were used to identify antigens recognised by randomly selected antibody fragments from a phage display antibody library [35]. Screening 12 different antibody fragments on an array of 27,000 expression clones, delivered four novel and highly specific antigen-antibody pairs. In a related approach, antibody arrays were used for the identification of specific antibody-producing bacteria [36]. For this purpose, bacteria containing phagemid selected from a phage antibody library by in vitro panning on chosen antigens were arrayed on filter membranes. After cell growth, antibody production was induced and specifically binding antibodies were captured and identified on a second, antigen-coated membrane. By screening 18,342 antibody clones at a time, highly specific antibodies were selected after just one round of panning.

In autoimmune diseases self-reacting antibodies, i.e., produced against the organism's own proteins and epitopes, play an important role in the clinical manifestation of the diseases. Therefore, the generation of an antibody profile of pa- 
tients with autoimmune disease is believed to be medically relevant and informative. Characterisation of autoimmune patient sera on protein chips would allow the diagnosis of autoimmune diseases based upon the presence of specific auto-antibodies. So far, for the identification of antigens recognised by auto-antibodies, sera were hybridised to uncharacterised gt $11 \mathrm{cDNA}$ phage libraries or to tissue extracts separated by $1 \mathrm{D}$ or $2 \mathrm{D}$ gel electrophoresis $[37,38]$. The following characterisation of the identified antigens is labour intensive, also requiring expensive sequencing of the found proteins. Such characterisation resulted in novel functions attributed to these proteins, which can then be used as potential therapeutical targets [39]. To simplify the characterisation of auto-antibodies, serum can be applied to protein arrays containing large numbers of recombinant proteins of known identity. Moreover, using protein arrays will overcome the problems associated with protein level variation in natural tissue extracts and hence increase reproducibility. The application of protein chips allows us to determine the binding profile of the autoimmune antibodies of each patient and for each disease. Once disease-specific antigens are known, it is possible to create a diagnostic protein array. As shown by Lueking et al., apparently specific monoclonal antibodies ( $\alpha$-HSP90, $\alpha$ - $\beta$-tubulin) showed considerable cross-reactivity with other proteins following incubation on protein microarrays, consisting of 96 in liquid bacterial cultures expressed proteins of the hEx1 library [19]. In a way, this is not surprising, as antibodies are not usually tested against whole libraries of proteins. However, in immunohistochemical or physiological studies against whole cells or tissue extracts, this cross-reactivity of antibodies can lead to false interpretations. Therefore, the characterisation of the binding specificity of antibodies used extensively in diagnostic tools is of prime importance.

\section{7}

\section{Outlook}

To achieve standardised microarrays carrying thousands of verified recombinant proteins, high-throughput methods for protein expression and purification are required. This has to be accompanied with a pipeline for the identification and verification of expression products. Initial experiments have shown that especially MALDI-MS is a powerful tool to monitor the quality of recombinant proteins. Combining protein expression and purification in array (microtitre plate) format with high-throughput protein mass determination by mass spectrometry leads to a large number of identified library clones and their corresponding expression products $[17,20]$. This approach can be used either to identify unknown clones from expression libraries or to verify expression products generated at high-throughput. In addition mass spectrometric data allows us to compare recorded spectra from recombinant and native proteins, which results in unambiguous protein identification in, e.g., $2 \mathrm{D}$ protein gels.

A very crucial part in protein array technology is played by the deposition of the proteins on a suitable surface. As described earlier, the nature of the surface with influencing parameters like charge, viscosity, pore size, $\mathrm{pH}$, binding capacity, unspecific protein binding, etc. is essential for the generation of protein arrays that contain the proteins in an biologically active shape and form. New sur- 
faces will lead to more native "living protein arrays". The basics for such a technology has been described by the analysis of protein-protein interactions in S. cerevisiae using the yeast two-hybrid screens in array format [11,31]. So called "living arrays" were constructed consisting of a nearly complete set of yeast open reading frames cloned as fusions with the Gal4 activation domain. This clone set was co-transformed with a set of putative interaction partners cloned as fusions to the Gal4 DNA binding domain and subsequently arrayed on filter membranes. Protein-protein interaction was detected by arraying of the co-transformed clone set on selective media. By screening 5,345 yeast open reading frame-Gal4 activation domain fusions with 195 Gal4 DNA binding domain fusions, 957 putative interactions, involving 1,004 yeast proteins, were identified. The recombinant expression of all open reading frames of Saccharomyces cerevisiae has already been achieved. A nearly complete collection of yeast strains for the expression of 6,144 open reading frames as fusion proteins was generated, divided in pools and screened for biological activities. Collections like this can form the basis of future protein microarrays by representing a large portion of gene products. As an example for an functional assay, 119 protein kinases were expressed, purified as GST fusion proteins, arrayed and cross-linked in a protein chip format and assayed for autophosphorylation by treatment with radiolabelled ATP. Also, substrate specificity was assayed with protein chips each carrying one of a set of kinase substrates. The kinases and the radiolabelled ATP were arrayed by pipetting onto the substrate coated surfaces and phosphorylation was monitored [40-42].

In addition to all array applications, clones and their recombinant proteins will form the basis for "structural genomics", a research field that aims to resolve the molecular structure of biomolecules and biomolecule complexes. Within the Berlin Protein Structure factory the same proteins that are deposited onto arrays are recombinantly expressed in yeast and E. coli and are subjects for crystallisation and the subsequent X-ray scattering as well as for NMR experiments. Similar technology to the technology described here is used with the immortal protein resources to do the probe preparation for structural genomics. One example is a high-precision sub-microlitre liquid dispensing system that has been developed for the preparation of hanging drop arrays for protein crystallisation. These arrays consist of $2 \mu \mathrm{L}$ to $100 \mathrm{~nL}$ drops and are used to screen for suitable buffer and salt conditions for protein crystallisation [25]. The technology developed for systematic data generation in genomics and proteomics will enable us to crystallise proteins that are available only in very tiny amounts and, therefore, contribute to our understanding of genome structure and physiological function.

\section{8}

\section{References}

1. Maier E, Meier-Ewert S, Bancroft D, et al (1997) Automated array technologies for gene expression profiling. Drug Discovery Today 2(8):315-324

2. Lander ES (1999) Array of hope. Nat Genet 21(1 Suppl):3-4

3. Meier-Ewert S, Lange J, Gerst H, et al (1998) Comparative gene expression profiling by oligonucleotide fingerprinting. Nucleic Acids Res 26(9):2216-2223

4. Schena M, Shalon D, Heller R, et al (1996) Parallel human genome analysis: microarraybased expression monitoring of 1000 genes. Proc Natl Acad Sci USA 93(20): 10614-10619 
5. Heller RA, Schena M, Chai A, et al (1997) Discovery and analysis of inflammatory diseaserelated genes using cDNA microarrays. Proc Natl Acad Sci USA 94(6):2150-2155

6. Khan J, Saal LH, Bittner ML, et al (1999) Expression profiling in cancer using cDNA microarrays. Electrophoresis 20(2):223-229

7. Khan J, Bittner ML, Chen Y, et al (1999) DNA microarray technology: the anticipated impact on the study of human disease. Biochim Biophys Acta 1423(2):M17-28

8. Eickhoff $\mathrm{H}$, Schuchhardt J, Ivanov I, et al (2000) Tissue gene expression profiling using arrayed normalized cDNA libraries. Genome Res 10(8):1230-1240

9. Schuchhardt J, Beule D, Malik A, et al (2000) Normalization strategies for cDNA microarrays. Nucleic Acids Res 28(10): E47

10. Walter G, Büssow K, Cahill D, et al (2000) Protein arrays for gene expression and molecular interaction screening. Curr Opin Microbiol 3(3):298-302

11. Emili AQ, Cagney G (2000) Large-scale functional analysis using peptide or protein arrays. Nat Biotechnol 18(4):393-397

12. Klose J, Kobalz U (1995) 2-dimensional electrophoresis of proteins - an updated protocol and implications for a functional-analysis of the genome. Electrophoresis 16(N6): $1034-1059$

13. Gauss C, Kalkum M, Lowe M, et al (1999) Analysis of the mouse proteome. (I) Brain proteins: separation by two-dimensional electrophoresis and identification by mass spectrometry and genetic variation. Electrophoresis 20(3):575-600

14. Frank R (1992) Spot synthesis: an easy technique for the positionally addressable parallel chemical synthesis on a membrane support. Tetrahedron 48:9217-9232

15. Heyman JA, Cornthwaite J, Foncerrada L, et al (1999) Genome-scale cloning and expression of individual open reading frames using topoisomerase I-mediated ligation. Genome Res 9(4):383-392

16. Büssow K, Cahill D, Nietfeld W, et al (1998) A method for global protein expression and antibody screening on high-density filters of an arrayed cDNA library. Nucleic Acids Res 26(21):5007-5008

17. Büssow K, Nordhoff E, Lübbert C, et al (2000) A human cDNA library for high-throughput protein expression screening. Genomics $65(1): 1-8$

18. Lueking A, Holz C, Gothold C, et al (2000) A system for dual protein expression in Pichia pastoris and Escherichia coli. Protein Expr Purif 20:372-378

19. Lueking A, Horn M, Eickhoff $\mathrm{H}$, et al (1999)Protein microarrays for gene expression and antibody screening. Anal Biochem 270(1):103-111

20. Egelhofer V, Büssow K, Luebbert C, et al (2000) Improvements in Protein Identification by MALDI-TOF-MS Peptide Mapping. Anal Chem 72(13):2741 - 2750

21. MacBeath G, Schreiber SL (2000) Printing proteins as microarrays for high-throughput function determination. Science 289(5485):1760-1763

22. Parinov S, Barsky V, Yershov G, et al (1996) DNA sequencing by hybridization to microchip octa-and decanucleotides extended by stacked pentanucleotides. Nucleic Acids Res 24(15):2998-3004

23. Arenkov P, Kukhtin A, Gemmell A, et al (2000) Protein microchips: use for immunoassay and enzymatic reactions. Anal Biochem 278(2):123-131

24. Schuerenberg M, Luebbert C, Eickhoff $\mathrm{H}$, et al (2000) Prestructured MALDI-MS sample supports. Anal Chem 72(15):3436-3442

25. Müller U, Nyarsik L, Horn M, et al (2001) Development of a technology for automation and miniaturisation of protein crystallisation. J Biotechnol (in press)

26. Sanders GHW, Manz A (2000) Chip-based microsystems for genomic and proteomic analysis. Trends Anal Chem 19(6):364-378

27. Cheung VG, Morley M, Aguilar F, et al (1999) Making and reading microarrays. Nat Genet 21(1 Suppl): $15-19$

28. Ge H (2000) UPA, a universal protein array system for quantitative detection of proteinprotein, protein-DNA, protein-RNA and protein-ligand interactions. Nucleic Acids Res 28(2): 3

29. Rogers KR (2000) Principles of affinity-based biosensors. Mol Biotechnol 14(2):109-129 
30. Merchant M, Weinberger SR (2000) Recent advancements in surface-enhanced laser desorption/ionization-time of flight-mass spectrometry. Electrophoresis 21(6):1164-1177

31. Uetz P, Giot L, Cagney G, et al (2000) A comprehensive analysis of protein-protein interactions in Saccharomyces cerevisiae. Nature 403(6770):623-627

32. Holt LJ, Enever C, de Wildt RM, et al (2000) The use of recombinant antibodies in proteomics. Curr Opin Biotechnol 11(5):445-449

33. Hoogenboom HR, de Bruine AP, Hufton SE, et al (1998) Antibody phage display technology and its applications. Immunotechnology 4(1):1-20

34. Collins J (1997) Phage Display. In: Moos WH, Pavia MR, Ellington A, Kay BK (eds) Annual Reports in Combinatorial Chemistry and Molecular Diversity, vol 1. Kluwer Academic Pub, pp $210-262$

35. Holt LJ, Büssow K, Walter G, et al (2000) By-passing selection: direct screening for antibodyantigen interactions using protein arrays. Nucleic Acids Res 28(15):E72

36. de Wildt RM, Mundy CR, Gorick BD, et al (2000) Antibody arrays for high-throughput screening of antibody-antigen interactions. Nat Biotechnol 18(9):989-994

37. Latif N, Baker CS, Dunn MJ, et al (1993) Frequency and specificity of antiheart antibodies in patients with dilated cardiomyopathy detected using SDS-PAGE and western blotting. J Am Coll Cardiol 22(5):1378-1384

38. Pohlner K, Portig I, Pankuweit S, et al (1997) Identification of mitochondrial antigens recognized by antibodies in sera of patients with idiopathic dilated cardiomyopathy by twodimensional gel electrophoresis and protein sequencing. Am J Cardiol 80(8):1040-1045

39. McCurdy DK, Tai LQ, Nguyen J, et al (1998) MAGE Xp-2: a member of the MAGE gene family isolated from an expression library using systemic lupus erythematosus sera. Mol Genet Metab 63(1):3-13

40. Joos TO, Schrenk M, Hopfl P, et al (2000) A microarray enzyme-linked immunosorbent assay for autoimmune diagnostics. Electrophoresis 21(13):2641-2650

41. Martzen MR, McCraith SM, Spinelli SL, et al (1999) A biochemical genomics approach for identifying genes by the activity of their products. Science 286(5442):1153-1155

42. Cohen CB, Chin-Dixon E, Jeong S, et al (1999) A microchip-based enzyme assay for protein kinase A. Anal Biochem 273(1):89-97

Received: June 2001 